\title{
Impact of climate change on outdoor thermal comfort in cities in united states
}

\author{
Yuqiao Huang ${ }^{1}$, Dayi Lai $^{2}$, and Yiqing Liu ${ }^{3,}$ Huang Xuan ${ }^{2, *}$ \\ ${ }^{1}$ School of Civil Engineering and Architecture, Zhejiang Sci-Tech University, Hangzhou, China 310018 \\ ${ }^{2}$ Department of Architecture, School of Design, Shanghai Jiao Tong University, Shanghai, China 200240 \\ ${ }^{3}$ Department of Architecture and Built Environment, University of Nottingham, Nottingham, UK NG7 2RD
}

\begin{abstract}
Since urban open spaces provide various benefits to the citizens, it is necessary to improve the outdoor thermal comfort in urban open spaces. However, global warming increases heat stress and at the same time decrease cold stress of outdoor spaces. The final impact of climate change on outdoor thermal comfort is not evident, and depends on the climate characteristics. This study investigated the influence of climate change on outdoor thermal comfort conditions of five selected cities (Minneapolis, New York City, San Francisco, Miami, and Las Vegas) with distinctive climate patterns in the United States. It is found that all cities suffered from deterioration in thermal comfort. This is because the increases in the heat stress rate were greater than the decreases in cold stress rate. In the $2080 \mathrm{~s}$, the greatest reduction in acceptable thermal stress rate happened in Miami from $44.7 \%$ to $21.3 \%$ under high emission scenario.
\end{abstract}

\section{Introduction}

With continuous urbanization, more than half of the world's population now lives in cities [1]. Urban open spaces play an important role in urban population's daily life because these spaces provide places for citizens' activity. The quality of urban open spaces largely depends on the thermal environment and the concomitant thermal comfort. Researches have shown that occupancy maximized under thermally comfortable condition [2]. However, a warming climate may deteriorate the outdoor thermal comfort. According to the projection of Intergovernmental Panel on Climate Change [3], the global mean surface air temperature will increase by 0.3 to $4.8{ }^{\circ} \mathrm{C}$ at the end of $21^{\text {st }}$ century, and other climatic variables, such as radiation and wind speed will also change. The changing climate imposes uncertainties on the thermal comfort condition of urban open spaces.

A few researchers have addressed the influence of climate change on outdoor thermal comfort. For example, Cheung and Hart found that the future climate in Hong Kong will shifted from "no thermal stress" to "moderate thermal stress and strong thermal stress" [4]. Thorsson et al. studied the future outdoor thermal comfort conditions in Gothenburg, Sweden [5]. They found the overall hours of "no thermal stress" increased because the decrease in strong/extreme cold stress time period is longer than that of strong/extreme heat stress. Since Hong Kong and Gothenburg have distinctive climate, the impact of climate change on outdoor thermal comfort for these two cities are totally different. The contrasting research findings suggest further studies on the influence of global warming on outdoor thermal comfort in more climate regions.

This study investigated the effect of climate change on outdoor thermal comfort in five cities with distinctive climate characteristics in the United States. The paper reports our findings.

\section{Methods}

\subsection{Studied cities}

In order to include variation in the climate, five representative cities from five major climate zones defined by Department of Energy of United States were selected for further investigation [6]. The five cities are Minneapolis from cold/very cold climate region, New York City from mixed-humid climate region, San Francisco from marine climate region, Miami from hothumid climate region, and Las Vegas from hot-dry/mixeddry climate region. The location of the five selected cities were indicated in Figure 1.

\footnotetext{
* Corresponding author: xuanh@sjtu.edu.cn 


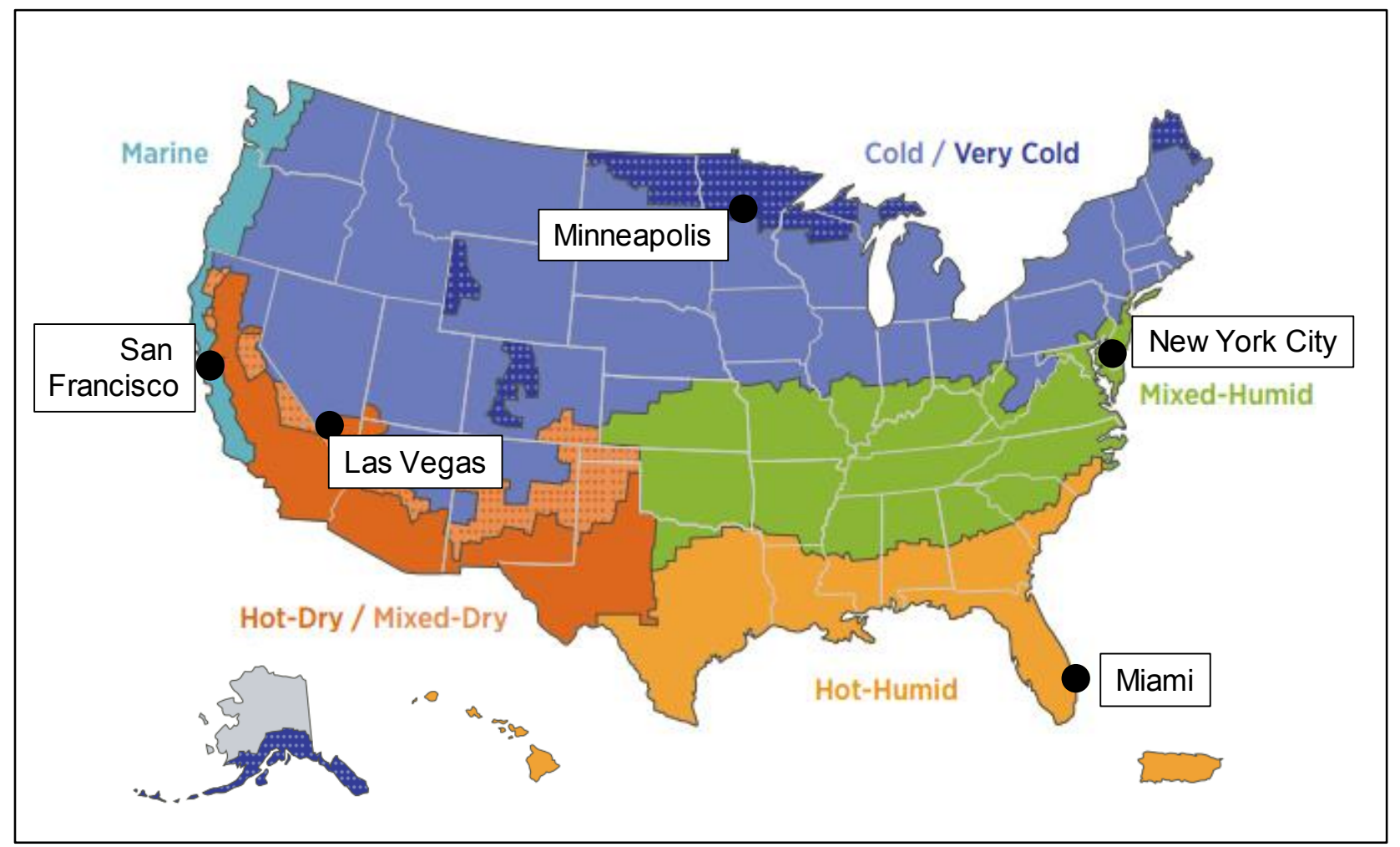

Figure 1. Studied cities in this investigation

\subsection{Generation of future weather data}

This study adopted the future weather data of cities in United States generated by Wang and Chen [7]. The HadCM3 model was used to project future climate change for three different $\mathrm{CO}_{2}$ scenarios: $\mathrm{A} 1 \mathrm{~F} 1, \mathrm{~A} 2$, and $\mathrm{B} 1$ as high, medium, and low emission scenarios [8]. HadCM3 offers the monthly change in dry-bulb temperature, relative humidity, wind speed, and solar radiation, which are the four main variables affecting outdoor thermal comfort and can be found on IPCC website for three emission scenarios. Since HadCM3 model only provides monthly data, a morphing method was applied to generate hourly climate data [7]. Typical Meteorological Year 3 (TMY3, from 1991-2005) dataset was used as baseline and was denoted as 1998, while the future TMY data for the 2050s and 2080s were generated for the evaluation of future outdoor thermal comfort.

\subsection{Determining outdoor thermal comfort}

Universal Thermal Climate Index was employed to evaluate the outdoor thermal comfort condition under current and future climate [9]. UTCI is defined as the equivalent air temperature of a typical environment that produce the same physiological responses for a standard person under an actual environment. The calculation of UTCI requires four climatic variables: the dry-bulb temperature, relative humidity, wind speed, and mean radiant temperature. The previous three variables were directly from the TMY datasets, the mean radiant temperature was calculated by RayMan model [10]. The site for calculation was considered as open ground without any block of solar radiation. Because occupants come to the urban open spaces mainly during the daytime, this study only used data from 7:00 a.m. to 18:00 p.m. of each day, and a total of 4380 hours were considered.

Table 1 shows the UTCI categorization used in this study. While the UTCI was lower than $0{ }^{\circ} \mathrm{C}$, the extreme, very strong, strong, and moderate cold stresses were combined as "unacceptable cold stress", the slight cold stress and no thermal stress categories from 0 to $26{ }^{\circ} \mathrm{C}$ UTCI were set as "Acceptable thermal stress", and over $26^{\circ} \mathrm{C}$ UTCI was further categorized as "unacceptable heat stress".

Table 1. UTCI thermal stress categories used in this study

\begin{tabular}{ccc}
\hline UTCI $\left({ }^{\circ} \mathrm{C}\right)$ & Stress category & $\begin{array}{c}\text { Further deduced } \\
\text { stress category }\end{array}$ \\
\hline$<-40$ & Extreme cold stress & \\
-40 to -27 & Very strong cold stress & $\begin{array}{c}\text { Unacceptable } \\
\text { cold stress }\end{array}$ \\
-27 to -13 & Strong cold stress & \\
-13 to 0 & Moderate cold stress & \\
\hline 0 to 9 & Slight cold stress & $\begin{array}{c}\text { Acceptable } \\
\text { thermal stress }\end{array}$ \\
9 to 26 & No thermal stress & \\
\hline 26 to 32 & Moderate heat stress & Unacceptable \\
32 to 38 & Strong heat stress & \\
38 to 46 & Very strong heat stress & \\
$>46$ & Extreme heat stress & \\
\hline
\end{tabular}




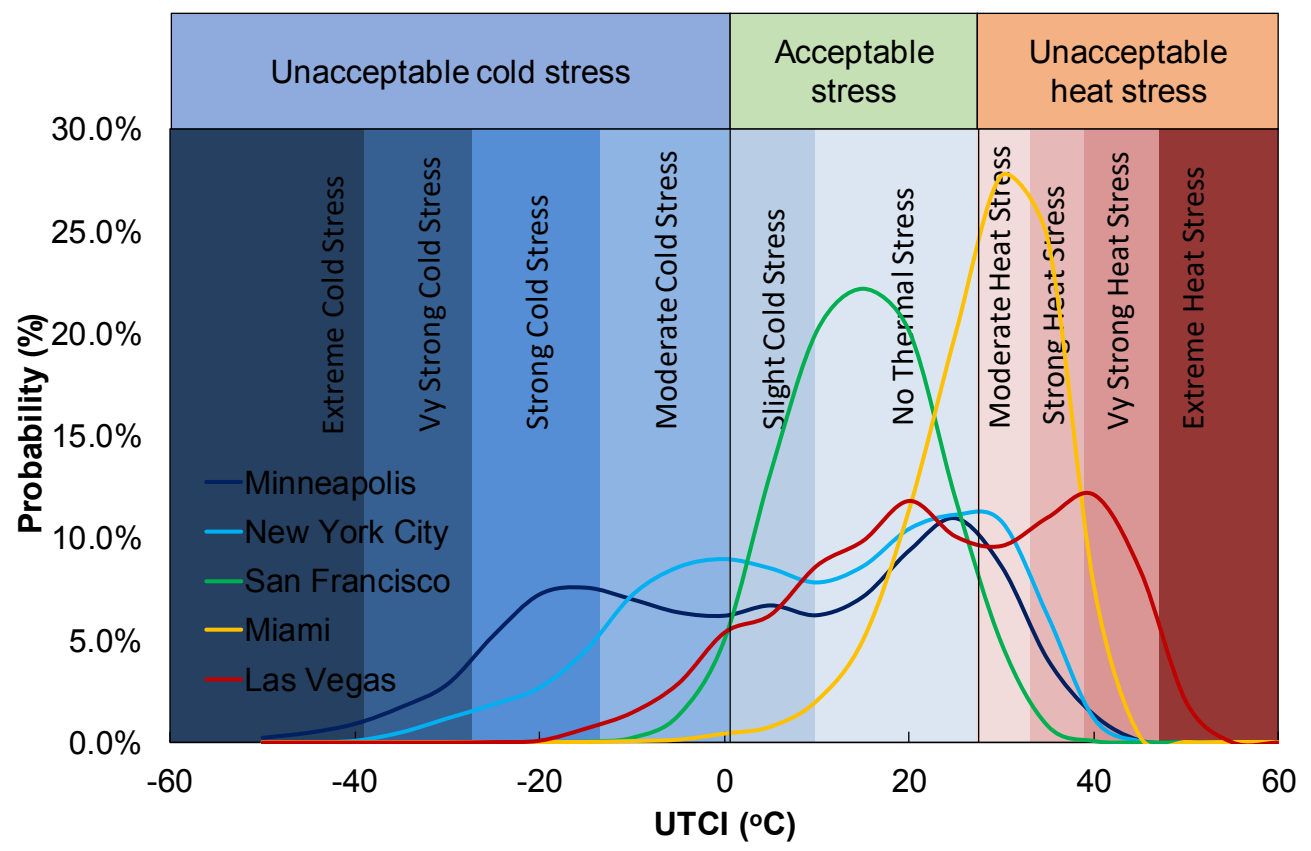

Figure 2. Probability distribution of UTCI calculated based on TMY3 data of each city

\section{Results}

This section first presents the current outdoor thermal comfort condition of the five cities as calculated based on TMY3 dataset. Then, the outdoor thermal comfort conditions in the 2050s and 2080s for different scenarios were depicted.

\subsection{Current situation}

Figure 2 shows the UTCI probability distribution for the five representative cities. Distinctive patterns of outdoor thermal comfort condition among cities were revealed. The probability distributions for Minneapolis, New York City, and Las Vegas were wider than those of San Francisco and Miami. The narrow outdoor thermal comfort ranges of San Francisco and Miami may be attributed to the coastal influence. The UTCI probability distribution for San Francisco was concentrated in the acceptable thermal stress range, while the Miami had a very high frequency of moderate and strong heat stress. Minneapolis had the lowest UTCI, with its lowest value under $-50{ }^{\circ} \mathrm{C}$, while Les Vegas had the highest UTCI of over $50^{\circ} \mathrm{C}$.

Table 2 summarizes the rates of unacceptable cold stress, acceptable thermal stress, and unacceptable heat stress of each city. Minneapolis had the highest unacceptable cold stress rate $(45.6 \%)$, followed by New York City (35.4\%). The unacceptable cold stress rate for the other three cities was generally lower than $10 \%$, with Miami seldom encountered any cold stress. However, unacceptable heat stress was as high as $54.7 \%$ in Miami. Las Vegas also had around $40 \%$ of unacceptable heat stress. Minneapolis and New York City also had over 10\% of unacceptable heat stress rate. San Francisco had the most pleasant outdoor thermal comfort condition with
$89.2 \%$ of acceptable thermal stress rate. The acceptable thermal stress rate for the other four cities were from $40 \%$ to $50 \%$.

Table 2. Comparison of unacceptable cold stress rate, acceptable thermal stress rate, and unacceptable heat stress rate of each city in existing scenario

\begin{tabular}{cccc}
\hline & $\begin{array}{c}\text { Unacceptab } \\
\text { le cold } \\
\text { stress rate }\end{array}$ & $\begin{array}{c}\text { Acceptable } \\
\text { thermal } \\
\text { stress rate }\end{array}$ & $\begin{array}{c}\text { Unacceptab } \\
\text { le heat } \\
\text { stress rate }\end{array}$ \\
\hline $\begin{array}{c}\text { Minneapolis } \\
\text { New York } \\
\text { City }\end{array}$ & $45.6 \%$ & $42.4 \%$ & $11.9 \%$ \\
San Francisco & $6.7 \%$ & $89.4 \%$ & $48.4 \%$ \\
Miami & $0.6 \%$ & $44.7 \%$ & $54.7 \%$ \\
Las Vegas & $10.5 \%$ & $48.5 \%$ & $39.8 \%$ \\
\hline
\end{tabular}

\subsection{Future scenarios}

To demonstrate the general trend of outdoor thermal comfort under climate change, this study calculated the annual mean UTCI for 1998, 2050s, and 2080s under various emission scenarios for each city. Figure 3 plots the results. Miami had the highest annual mean UTCI, followed by Las Vegas, San Francisco, New York City, and Minneapolis. For every case, the annual UTCI increased, even for the low emission scenario. The degree of increase ranged from 2.7 to $4.4 \mathrm{~K}$ for the low emission scenario. For the high emission scenario, the highest rise in UTCI can be $8.5 \mathrm{~K}$ (Minneapolis). The increases for New York City, San Francisco, Miami, and Las Vegas were $7.3 \mathrm{~K}, 5.6 \mathrm{~K}, 5.2 \mathrm{~K}$, and $7.5 \mathrm{~K}$, respectively. 

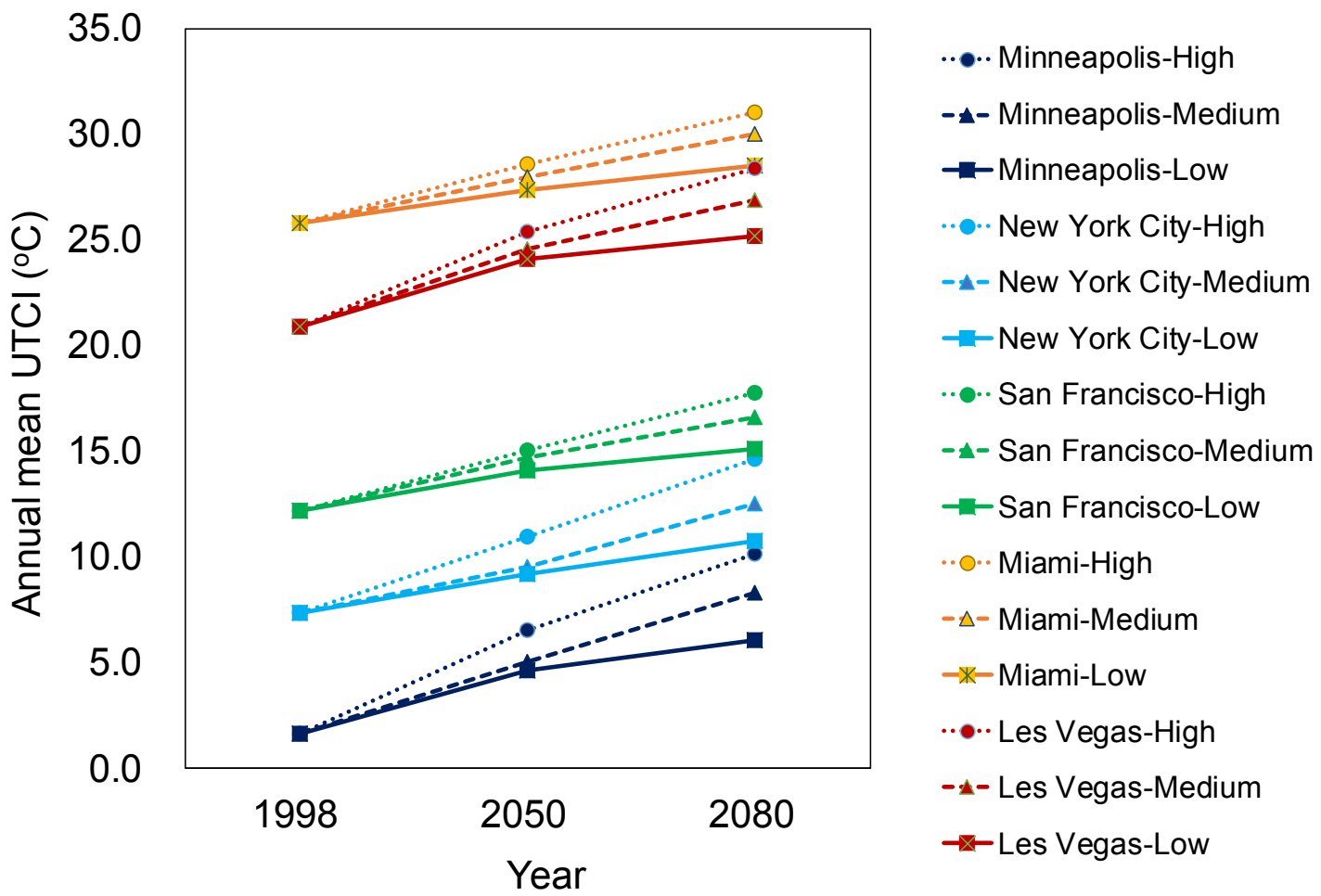

Figure 3. Changes in annual mean UTCI for each city under various emission scenarios

Figure 4 shows the overall changes in the unacceptable cold stress rate, acceptable thermal stress rate, and unacceptable heat stress rate calculated for the five selected cities under various emission scenarios. Generally speaking, higher emission led to greater change in outdoor thermal comfort condition. From Figure 4(a), the unacceptable cold stress rate decreased for all cities, but the reductions were different among cities. For Minneapolis and New York City, the reduction can be as much as $10 \%$ under high emission scenarios. For Miami, since very few cold stress existed, the unacceptable cold stress rate did not have a much change. However, Miami witnessed a significant increase in the unacceptable heat stress from $54.7 \%$ to $78.6 \%$ under high emission scenario.

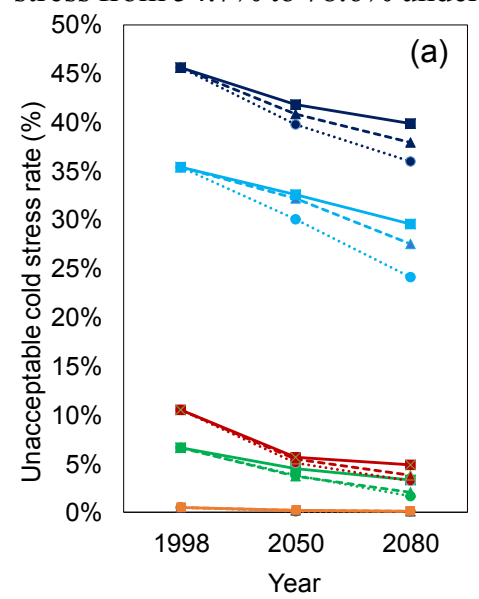

Other cities also experience large increase in heat stress rate.

Because the increase in unacceptable heat stress rate exceeded the decrease in unacceptable cold stress rate, the overall acceptable thermal stress rate reduced. Miami suffered the most substantial deterioration in outdoor thermal comfort among all cities. Under high emission scenario, the acceptable thermal stress rate decreased from $44.7 \%$ to $21.3 \%$. This may be attributed to the fact that the Miami does not have any cold stress and the global warming only increased the heat stress without any compensation for the decrease in cold stress. For other cities, the reduction in acceptable thermal stress rate ranged from $5 \%$ to $10 \%$.

Figure 4. Changes in (a) unacceptable cold stress, (b) acceptable thermal stress, and (c) unacceptable heat stress for each city under different emission scenarios 


\section{Discussion}

The reduction in acceptable thermal stress rate in all studied cities highlights the necessity of taking action to mitigate the consequences brought by climate change. According to the analysis results of Figure 4, the increases in heat stress is a major problem, especially for hot climate cities such as Miami and Las Vegas. Various passive design strategies, such as shading, adding vegetation, using reflective surfaces [11], to reduce the heat stress should be considered.

Actual urban outdoor spaces have various geometry, and thus the resulting thermal radiation field will vary greatly. To ensure comparability, the assessment of outdoor thermal comfort was conducted for totally open places with Sky View Factor (SVF) equals unity. However, such assumption may lead to unrealistic results with overestimated thermal stress. In future studies, it is necessary to investigate the thermal comfort in various realistic urban spaces with varying SVF.

\section{Conclusions}

Climate change is a critical factor that influence the future outdoor thermal comfort condition, and the impact is different for various climate regions. This study targeted at five representative cities with distinctive climatic characteristics in the United States: Minneapolis, New York City, San Francisco, Miami, and Las Vegas. The future weather data were generated for every hour by morphing the monthly weather data offered by HadCM3 model under high, medium, and low emission scenarios. The generated weather data were used to calculate the UTCI index for the future outdoor thermal comfort analyses. The analyses lead to the following conclusions:

1. Under current situation, San Francisco had the most pleasant climate, with $89.2 \%$ of acceptable thermal stress rate. The acceptable thermal stress rate for other four cities were between $40 \%$ and $50 \%$.

2. The annual mean UTCI increased with time. Higher emission led to greater increase in UTCI. Under high emission scenario, the rise in annual mean UTCI in studied cities ranged from $5.2 \mathrm{~K}$ to $8.5 \mathrm{~K}$.

3. For all cities, although the cold stress decreased, the increase in heat stress is greater than the decrease in cold stress. As a result, the overall acceptable thermal stress decreased. The greatest decrease occurred at Miami under high emission scenario, from $44.7 \%$ to $23.4 \%$.

This research was supported by Shanghai Sailing Program of Science and Technology Commission of Shanghai Municipality through Grant No. 19YF1424000.

\section{References}

1. United Nations, World Urbanization Prospects (2014) https://esa.un.org/unpd/wup/publications/files/wup2 014-highlights.Pdf/ Accessed 1 December 2017.

2. D. Lai, C. Zhou, J. Huang, Y. Jiang, Z. Long, Q. Chen, Energy Build. 68, 713 (2014)

3. Intergovernmental Panel on Climate Change (IPCC), Climate Change 2013: The Physical Basis Technical Summary (2013), http://www.climatechange2013.org/images/report/ WG1AR5_ALL_FINAL.pdf.

4. C.S.C. Cheung, M.A. Hart, Int. J. Biometeorol. 58(2), 137 (2014)

5. S. Thorsson, F. Lindberg, J. Björklund, B. Holmer, D. Rayner, Int. J. Climatol. 31(2), 324 (2011)

6. United States Department of Energy, Building , America Climate-Specific Guidance (2019), https://www.energy.gov/eere/buildings/buildingamerica-climate-specific-guidance

7. H. Wang, Q. Chen, Energy Build. 82,428 (2014)

8. S. Solomon, D. Qin, M. Manning, Z. Chen, M. Marquis, K.B. Averyt, M. Tignor, H.L. Miller, Climate Change 2007: The Physical Science Basis (2007)

9. G. Jendritzky, R. De Dear, G. Havenith, Int. J. Biometeorol. 56(3), 421 (2012)

10. A. Matzarakis, F. Rutz, H. Mayer, Biometeorol. 51(4), 323 (2007)

11. D. Lai, W. Liu, T. Gan, K. Liu, Q. Chen, Sci. Total Environ. 661, 337 (2019) 